\title{
Geohelmintiasis e hiper-IgE en escolares de un área rural y un área urbana de Honduras entre septiembre 2014 a junio 2015
}

\author{
Carol Anahelka Rodríguez, Maria Mercedes Rueda, \\ Maritza Canales, Gabriela María Matamoros, Joel Saamir García ${ }^{1}$ \\ Antonio Gabrie, Lourdes Sánchez ${ }^{2}$
}

\section{RESUMEN}

Introducción: Los helmintos transmitidos por el suelo (geohelmintos) son parásitos intestinales que polarizan el sistema inmune hacia una respuesta caracterizada por altos valores circulantes de Inmunoglobulina $\mathrm{E}(\mathrm{lg} E)$. Honduras no tiene datos recientes que demuestren este fenómeno biológico. Objetivos: Determinar la asociación entre infecciones por geohelmintos con hiper-lgE, comparando escolares de una zona rural y una urbana de Honduras. Metodología: Estudio descriptivo transversal. Se seleccionaron dos comunidades, una con alta prevalencia de geohelmintos $(>50 \%)$ y otra con baja prevalencia de geohelmintos $(<20 \%)$. Se realizaron entrevistas a los participantes para identificar su edad e historia de desparasitación. Para determinar la prevalencia de parásitos se recolectaron muestras de heces y se procesaron con el método de Kato- Katz y Acetato de etilo. Para determinar los niveles de lgE sérica se tomó muestras sanguíneas y el suero se procesó utilizando la tecnología MagPIX® (kit de Bio-Plex Pro_IgE humana). De acuerdo con este método el límite de detección para la lgE sérica es de $0.040 \mathrm{ng} / \mathrm{ml}$. Aprobación ética CEI/MEIZ. Análisis estadístico paramétrico y no paramétrico.Resultados: Se estudiaron 73 niños del área rural y 71 del área urbana, de los cuales 35 (47.9\%) y 5 (7\%) estaban parasitados con una o más especie de geohelmintos (Trichuris trichiura, Ascaris lumbricoides, Ancylostomatídeos), respectivamente. Ocho participantes $(<6 \%)$ presentaron infecciones moderadas a severas. La población rural presentó 94.5\% (69) de hiper-lgE en contraste con la población urbana con 63.5\% (45), ( $p<0,001)$.Limitaciones: no se cuenta con antecedentes de procesos alérgicos relacionados con lgE.

Conclusiones: Se logró determinar que existe una diferencia significativa en los

\footnotetext{
${ }^{1}$ Beneficiarios de una beca sustantiva de la DICYP. Grupo de Investigación en Parasitología. Profesores de la Escuela de Microbiología, Facultad de Ciencias, UNAH: carol.rodriguez@unah.edu.hn; ma.mercedesrueda@yahoo.com; macg@unah.edu.hn

2 Grupo de Investigación en Parasitología. Department of Community Health Sciences, Brock University, St. Catharines, Ontario, Canada.
} 
niveles de $\lg E$ de los niños parasitados con geohelmintos entre la población rural y urbana. Esto puede explicar la disminución de reacciones alérgicas en las poblaciones mayormente expuestas a estos parásitos, lo cual podría comprobarse realizando estudios de IgE específica para los HTS.

Palabras clave: helmintos, IgE.

\section{ABSTRACT}

Introduction: Helminths transmitted by the soil (geohelminths) are the intestinal parasites that provoke the polarization of the immune system towards a response characterized by the high circulating values of Immunoglobulin $E(\mathrm{lgE})$. In Honduras, there are no recent studies demonstrating this biological phenomenon.Objectives: This study aims to determine the association between geohelminth infections with hyper$\operatorname{lgE}$, comparing schoolchildren from a rural area and an urban area of Honduras. Methodology: Descriptive transversal study. Two communities were selected, with a high prevalence of geohelminths (> 50\%) and an urban with low prevalence of geohelminths $(<20 \%)$. Interviews were conducted with the participants to determine their age, grade and history of deworming. To determine the prevalence of parasites, faecal samples were collected and processed with the Kato-Katz Method and Ethyl Acetate. To determine serum lgE levels, blood samples were taken, and the serum was processed using MagPIX® technology (human Bio-Plex Pro_lgE kit). According to this method the limit of detection for lgE is $0.040 \mathrm{ng} / \mathrm{ml}$. Ethical approval CEI/MEIZ. Parametric and non-parametric statistical analysis. Results: 73 children from the rural area and 71 from the urban area were studied, of which $35(47.9 \%)$ and $5(7 \%)$ were parasitized with one or more species of geohelminths (Trichuris trichiura, Ascaris lumbricoides, hookworm) respectively. Eight participants $(<6 \%)$ had moderate to severe infections. The rural population presented $94.5 \%$ (69) of hyper-lgE in contrast to the urban population with $63.5 \%(45),(p<0.001)$. Limitations: there is no history of allergic processes related to $\lg \mathrm{E}$.

Conclusions: This study was able to determine that there is a significant difference in the lgE levels of children with geohelminth parasites between rural and urban populations. This may explain the decline in allergic reactions in older populations.

Key words: helminths, IgE. 


\section{INTRODUCCIÓN}

Los geohelmintos son los parásitos más frecuentes entre todas las infecciones helmínticas humanas, estimándose que a nivel global infectan aproximadamente 2 mil millones de personas, especialmente niños (WHO, 2012). Los geohelmintos incluyen Ascaris lumbricoides, Trichiuris trichiura, ancylostomatídeos (Necator americanus y Ancylostoma duodenale) y Strongyloides stercoralis. Las geohelmintiasis agudas, especialmente cuando son infecciones mixtas o masivas, pueden resultar en una alta morbilidad. Sin embargo, el impacto en la salud infantil es generalmente causado por la naturaleza insidiosa y crónica de estas infecciones, conllevando a la malnutrición, retraso del crecimiento y deterioro de la función cognitiva (Cooper et al., 2015). Otros efectos fisiológicos de estas infecciones es la alteración de la respuesta inmunológica, la cual se modula disminuyendo la reacción contra los parásitos para evitar daño tisular e inflamación en el huésped. Ala vez, dicha modulación permite las infecciones crónicas y aumenta la vulnerabilidad a otras infecciones (Alcantara-Neves et al., 2014; Zaph, Cooper, \& Harris, 2014).

Otra alteración de la respuesta inmunológica ante presencia de geohelmintos en el intestino del huésped se manifiesta con altos niveles de lgE policlonales, observados en pobladores de áreas endémicas. La eosinofilia es causada por estimulación no específica como resultado de un mecanismo dependiente de Interleucina-4 (Belhassen-García et al., 2014; Cooper et al., 2008; Fitzsimmons, Falcone, \& Dunne, 2014). La respuesta basada en IgE está implicada en la protección del huésped contra el parásito, la cual se conoce como respuesta IgE-Th2 de los linfocitos-eosinófilos (Allen \& Wynn, 2011; McSorley \& Maizels, 2012). Curiosamente, varios estudios muestran que la presencia de infecciones por helmintos en los niños está inversamente asociada con un aumento de las respuestas de lgE a los alérgenos ambientales (Lau \& Matricardi, 2006). Sin embargo, en estudios realizados en países en desarrollo, la presencia de anticuerpos lgE frente a alérgenos no siempre ha sido asociada a enfermedades alérgicas, especialmente en áreas donde las infecciones por helmintos eran frecuentes.

Las causas de los altos niveles circulantes de lgE en las poblaciones tropicales pobres han sido poco estudiadas, pero se han atribuido a eczema atópico, asma y rinitis alérgica. También se postula que hay factores genéticos que predisponen la eosinofilia (Djuardi et al., 2013; Figueiredo et al., 2012).

Con el fin de conocer el comportamiento de la lgE en Honduras, se realizó el presente 
estudio en dos comunidades con diferentes prevalencias de geohelmintos, para realizar una comparación de los niveles de lgE entre aquellos individuos mayormente expuestos con los menormente expuestos.

\section{MATERIALES Y MÉTODOS}

\section{Tipo de estudio}

Estudio descriptivo transversal.

\section{Población}

Los estudios de campo se llevaron a cabo en agosto de 2015 en dos comunidades, el pueblo de Santa Cruz del Junco (área rural), en el municipio de Tela, Atlántida ubicado en el norte de Honduras y la ciudad de Tegucigalpa (área urbana), en el departamento de Francisco Morazán, ubicada en el centro de Honduras. Santa Cruz del Junco se ubica en la región ecológica II donde la prevalencia de geohelmintos es $>50 \%$ y Tegucigalpa en la región ecológica IV donde la prevalencia de geohelmintos es $\leq 20 \%$ (Mejia Torres et al., 2014). Se incluyeron niños de 7 a 15 años, 73 de la escuela "Celestino Canales Sierra" de Santa Cruz del Junco y 71 de la escuela "Villa Olímpica" de Tegucigalpa.

\section{Recolección de datos}

Los datos demográficos fueron recolectados usando un cuestionario cara a cara de cinco minutos. Los datos obtenidos incluyen edad, género, grado escolar, historia de infecciones por parásitos y desparasitación.

\section{Recolección de muestras}

Se recolectó una única muestra de heces de los participantes para determinar la presencia e intensidad de la infección por geohelmintos utilizando la técnica de Kato Katz, según la recomendación de la OMS (WHO, 2012), con una plantilla de 41,7 mg (Vestergaard Frandsen SA). Estas muestras fueron traídas para los participantes el mismo día de recolección de la muestra. Las muestras fueron transportadas en envase frío al laboratorio clínico del Hospital Tela de Santa Cruz del Junco y al laboratorio de parasitología de la Escuela de Microbiología (UNAH) de Tegucigalpa. 
Se tomó una muestra única de sangre total de los participantes con punción venosa utilizando el sistema Vacutainer ${ }^{\mathrm{TM}}$ recogido en tubos de plástico sin anticoagulante. El suero se separó y se mantuvo a $-20^{\circ} \mathrm{C}$, para determinar los niveles de lgE total circulante usando la tecnología MagPIX®. Este procedimiento se realizó en el Laboratorio de Ciencias, Complejo de Cairns de la Universidad de Brock, Canadá.

\section{Procedimientos de laboratorio}

Las infecciones e intensidad de los geohelmintos se determinaron el mismo día de recogida en la tarde, tomando un tiempo de cuatro a seis horas. La preparación de Kato Katz se leyó entre 20 y 30 minutos después de la preparación. Los huevos de geohelmintos fueron identificados por sus rasgos característicos y contados sistemáticamente para estimar el número de huevos por gramo de heces (EPG), para clasificar las infecciones como intensidad ligera, moderada o severa, usando los criterios de la OMS (WHO, 2012). Para el control de la calidad, el 10\% de los frotis positivos y $100 \%$ de los negativos fueron releídos por un investigador diferente inmediatamente después de la primera lectura.

Las concentraciones séricas de IgE total también se cuantificaron utilizando una plataforma de perlas magnéticas, utilizando un kit singleplex, Bio-Plex Pro_Human IgE Isotyping (Laboratorios Bio- Rad, Inc. Hercules, CA, EE.UU.). El resultado obtenido se multiplicó por 24 para obtener valores en U/L. La hiper-IgE se definió como una concentración sérica superior a $100 \mathrm{Ul} / \mathrm{mL}$ y se clasificó como leve (>100-399 Ul/mL), moderada (>399-999 UI/mL) o grave ( $\geq 1000 \mathrm{UI} / \mathrm{mL}$ ) (Belhassen-Garcia et al., 2014). Se realizaron las pruebas de $\lg E$ de acuerdo con las instrucciones del fabricante (Bio-Rad laboratories, Inc. Hercules, CA, EE.UU.).

\section{Análisis estadístico}

La población del estudio se caracterizó mediante estadística descriptiva (frecuencia, porcentajes, medias y DE). Se calcularon las prevalencias puntuales para las infecciones totales y por especie de geohelmintos.

Debido a la distribución no Gaussiana de los niveles de lgE, se utilizaron métodos no paramétricos como sigue. Se calcularon las medias geométricas y el IC del $95 \%$. Se utilizó la prueba de T-Student para evaluar las diferencias entre los grupos. Todos los análisis estadísticos se llevaron a cabo utilizando Stata 13 (StataCorp LP, TX, EE.UU.) y el nivel de significancia se definió como $p<0,05$. 


\section{Consideraciones éticas}

Este estudio recibió la Autorización de Ética para la Investigación de Participantes Humanos de la Universidad de Brock (número de registro 14-224 - SANCHEZ, de fecha 5 de agosto de 2015), así como del Comité de Ética en Investigación del Programa de Maestría en Enfermedades Infecciosas y Zoonóticas, Microbiología, Universidad Nacional Autónoma de Honduras (número CEI-MEIZ 01- 2015, fechado el 1 de agosto de 2015). El consentimiento escrito e informado de los padres y el consentimiento de los niños para participar en el estudio se tomó 3 días antes de la encuesta. Un padre o un maestro estaban presentes mientras se realizaba la encuesta y se tomaban muestras.

\section{RESULTADOS}

144 escolares del primer al sexto grado (7 a 15 años) participaron en el estudio. 73 de la escuela "Celestino Canales Sierra" de Santa Cruz del Junco, en la ciudad de Tela y 71 de la escuela "Villa Olímpica" de Tegucigalpa. Cerca de la mitad de las poblaciones del estudio eran niñas. Con excepción de dos niños del grupo de Tegucigalpa, el resto reportó habían recibido tratamiento desparasitante en los últimos seis meses (cuadro 1).

Cuadro 1. Historia de parasitosis de acuerdo con el área estudiada.

\begin{tabular}{|l|c|c|}
\hline & $\begin{array}{c}\text { Tela, } \mathbf{n} \\
(\%)\end{array}$ & $\begin{array}{c}\text { Tegucigalpa, } \mathbf{n} \\
(\mathbf{\%})\end{array}$ \\
\hline Edad, media en años (DE) & $9.9(2.2)$ & $9.8(1.5)$ \\
\hline Niñas & $37(50.7)$ & $34(47.9)$ \\
\hline Historia de parasitosis* & & \\
\hline Presencia & $51(69.8)$ & $40(56.3)$ \\
\hline Historia de desparasitación ${ }^{+}$ & & \\
\hline Desparasitados & $73(100)$ & $68(93.1)$ \\
\hline No desparasitados & $0(0)$ & $2(2.8)$ \\
\hline
\end{tabular}

* ¿Alguna vez tuvo lombrices?

+ ¿Ha recibido tratamiento para las lombrices en los últimos 6 meses? 
Los resultados cualitativos de Kato-Katz fueron 100\% correlacionados con los de formol-acetato de etilo. La prevalencia general de geohelmintos fue $47,9 \%$ (35) en Tela y $7 \%$ (5) en Tegucigalpa (sólo T. trichiura). La prevalencia de T. trichiura A. lumbricoides y ancylostomatídeos en Tela fue de $41,1 \%$, (30), 13,7\% (10) y $11 \%$ (8), respectivamente. Las infecciones mixtas de geohelmintos representaron el $34.3 \%$ (12) de todas las infecciones observadas. El $80 \%$ (24) de los casos de tricuriasis en Tela fue de intensidad leve, y sólo uno de los cinco casos en Tegucigalpa fue moderado a severo $(20 \%)$, nueve $(90 \%)$ de los casos de ascariasis fue de intensidad leve (cuadro 2).

Cuadro 2. Prevalencia e intensidad de la infección de los geohelmintos por área.

\begin{tabular}{|l|c|c|c|}
\hline & $\begin{array}{c}\text { Tela } \\
\mathbf{n}(\%)\end{array}$ & $\begin{array}{c}\text { Tegucigalpa } \\
\mathbf{n}(\%)\end{array}$ & Valor $\mathbf{p}^{*}$ \\
\hline $\begin{array}{l}\text { Prevalencia total de infecciones } \\
\text { por geohelmintos }\end{array}$ & $35(47.9)$ & $5(7)$ & $<0.001$ \\
\hline Ascaris lumbricoides & $10(13.7)$ & $0(0)$ & 0.001 \\
\hline Trichuris trichiura & $30(41.1)$ & $5(7)$ & $<0.001$ \\
\hline Ancylostomatídeos & $8(11.0)$ & $0(0)$ & $<0.001$ \\
\hline Infecciones mixtas & $12(34.3)$ & $0(0)$ & $<0.001$ \\
\hline Intensidad de la infección & & & \\
\hline A. lumbricoides mod-severa & $1(10)$ & $0(0)$ & \\
\hline T. trichiura mod-severa & $6(20)$ & $1(20)$ & \\
\hline Ancylostomatídeos mod severa & $0(0)$ & $0(0)$ & \\
\hline
\end{tabular}

*Valor $p$ fue calculado con t-student

El cuadro 3 resume los niveles de $\lg E$ en ambas ciudades. La prevalencia de hiper-IgE en Tela fue del $94.5 \%$ (69) de estos, 36 (53\%) fueron severos, mientras que la hiper-lgE leve y moderada representó el $21 \%$ de cada uno. En Tegucigalpa, la prevalencia de hiper-lgE fue del $63 \%$ (28), la mayoría de los casos fueron leve $(30 \%)$, mientras que la hiper-lgE moderada y grave, representaron el $18.3 \%$ y el $15.4 \%$ respectivamente. Hubo diferencias significativas entre los grupos ( $\mathrm{Cl} 95 \%, \mathrm{p}>0,001)$. 
Cuadro 3. Niveles de lgE sérico de los participantes por área de estudio.

\begin{tabular}{|l|l|c|c|}
\hline & $\begin{array}{c}\text { Tela } \\
(\mathrm{n}=73)\end{array}$ & $\begin{array}{c}\text { Tegucigalpa } \\
(\mathrm{n}=71)\end{array}$ & Valor $\mathbf{p}^{*}$ \\
\hline $\begin{array}{l}\text { IgE, mediana en UI/mL } \\
\text { (Rango intercuantil) }\end{array}$ & $\begin{array}{c}1216.9 \\
(377.0-3136.8)\end{array}$ & $\begin{array}{c}168.9 \\
(63.4-657.4)\end{array}$ & $<0.001$ \\
\hline Hiper $\lg \mathrm{E}^{+} \mathrm{n},(\%)$ & $69(94.5)$ & $45(63.4)$ & $<0.001$ \\
\hline
\end{tabular}

*Valor p calculado con t-student

+Definido como valores $>100 \mathrm{UI} / \mathrm{L}$

\section{DISCUSIÓN}

Los hallazgos parasitológicos de este estudio demuestran un alta (47,9\%) prevalencia de geohelmintos en Tela y una baja prevalencia $(7 \%)$ en Tegucigalpa. Trichuris trichiura, Ascaris lumbricoides y ancylostomatídeos fueron identificados en Tela, sin embargo, en Tegucigalpa solo se confirmó la presencia de Trichuris trichiura. Los estudios anteriores realizados en Tela confirman que la prevalencia en esta zona es mayor al 30\% (Mejía Sánchez et al., 2014).

En el presente estudio se determinaron los niveles de lgE sérica, donde el $94.5 \%$ de los escolares de la zona rural (Tela) presentó un nivel hiper lgE (nivel de lgE> 100 $\mathrm{U} / \mathrm{mL}$ ), mientras que en la zona urbana (Tegucigalpa) sólo el $63.5 \%$ presentó hiper $\lg \mathrm{E}$. Existe una diferencia significativa $(p<0,001)$ entre los datos obtenidos en las zonas rurales y urbanas, lo que apoya los diferentes estudios que afirman que la exposición constante a estos parásitos puede observarse con un alto grado de hiperIgE en la población (Belhassen-García et al., 2014; Cooper et al., 2016).

En un estudio realizado en Nigeria, los escolares con infecciones por helmintos tenían niveles más altos de lgE (Arinola et al., 2015), igualmente Cooper y col. (Cooper et al., 2008) demostraron que los niveles de lgE disminuyeron en niños que recibieron tratamiento antihelmíntico; sin embargo en este estudio se presenta un alto grado desparasitación con altos niveles de lgE circulante, lo cual podría explicarse a la constante exposición al parásito. Todos estos hallazgos demuestran que la lgE puede ser un indicador de la infección por este tipo de parásitos.

En Honduras, un estudio de Gabrie y col., reveló una sorprendentemente alta preva- 
lencia de hiper- $\lg E$ entre los niños estudiados. También destacó una asociación significativa entre las infecciones por goehelmintos y el aumento de los niveles de lgE total (Gabrie, Rueda, Rodríguez, Canales, \& Sánchez, 2016).

En conclusión, este estudio mostró una diferencia significativa $(\mathrm{Cl} 95 \%, \mathrm{p}>0.001)$ en los niveles de lgE entre la población rural y urbana, siendo la población rural (con mayor exposición a los geohelmintos) la que presenta más casos de hiper-lgE. Además, se recomienda realizar estudios longitudinales y determinar lgE específica para una mejor comprensión de los hallazgos.

Se recomienda hacer pruebas de lgE específica para determinar a qué especie de helminto está dirigida esta respuesta, ya que la lgE específica contra Ascaris predispones a sibilancias y alergias ectópicas porque reaccionan cruzadamente con ácaros y cucarachas. Además, es necesario conocer la historia clínica relacionada con procesos alérgicos que pueden desencadenar este tipo de respuesta.

\section{AGRADECIMIENTOS}

Los autores del proyecto agradecen a todos los niños que participaron en este estudio, así como a sus padres, y autoridades escolares de Tela y Tegucigalpa. Este estudio recibió apoyo financiero de la UNAH mediante una subvención interna patrocinada por la Dirección de Investigación científica y Posgrados (DICyP) y a Brock University por su aporte en la realización de las pruebas inmunológicas.

\section{REFERENCIAS BIBLIOGRÁFICAS}

Alcantara-Neves, N. M., de, S. G. B. G., Veiga, R. V., Figueiredo, C. A., Fiaccone, R. L., da Conceicao, J. S. Barreto, M. L. (2014). Effects of helminth co-infections on atopy, asthma and cytokine production in children living in a poor urban area in Latin America. BMC Res Notes, 7, 817. doi: 10.1186/1756-0500-7-817

Allen, J. E., \& Wynn, T. A. (2011). Evolution of Th2 immunity: a rapid repair response to tissue destructive pathogens. PLoS Pathog, 7(5), e1002003. doi: 10.1371/journal.ppat. 1002003

Arinola, G. O., Morenikeji, O. A., Akinwande, K. S., Alade, A. O., Olateru-Olagbegi, O., Alabi, P. E., \& Rahamon, S. K. (2015). Serum Levels of Cytokines and lgE in Helminth-Infected 
Nigerian Pregnant Women and Children. Ann Glob Health, 81(5), 689-693. doi: 10.1016/j.aogh.2015.12.008

Belhassen-Garcia, M., Pardo-Lledias, J., Pérez del Villar, L., Muro, A., Velasco-Tirado, V., Blázquez de Castro, A., Cordero-Sanchez, M. (2014). Relevance of eosinophilia and hyper-IgE in immigrant children. Medicine (Baltimore), 93(6), e43. doi: 10.1097/MD.0000000000000043

Cooper, P. J., Alexander, N., Moncayo, A. L., Benítez, S. M., Chico, M. E., Vaca, M. G., \& Griffin, G.E. (2008). Environmental determinants of total lgE among school children living in the rural Tropics: importance of geohelminth infections and effect of anthelmintic treatment. [Randomized Controlled Trial Research Support, Non-U.S. Gov't]. BMC Immunol, 9, 33. doi: 10.1186/1471-2172-9-33

Cooper, P. J., Amorim, L. D., Figueiredo, C. A., Esquivel, R., Tupiza, F., Erazo, S., Barreto, M. L. (2015). Effects of environment on human cytokine responses during childhood in the tropics: role of urban versus rural residence. World Allergy Organ J, 8(1), 22. doi: 10.1186/s40413- 015-0071-2

Cooper, P. J., Chico, M. E., Amorim, L. D., Sandoval, C., Vaca, M., Strina, A. Strachan, D. P. (2016). Effects of maternal geohelminth infections on allergy in early childhood. J Allergy Clin Immunol, 137(3), 899-906 e892. doi: 10.1016/j.jaci.2015.07.044

Djuardi, Y., Supali, T., Wibowo, H., Kruize, Y. C., Versteeg, S. A., van Ree, R. Yazdanbakhsh, M. (2013). The development of TH2 responses from infancy to 4 years of age and atopic sensitization in areas endemic for helminth infections. Allergy Asthma Clin Immunol, 9(1), 13. doi: 10.1186/1710-1492-9-13

Figueiredo, J. P., Oliveira, R. R., Cardoso, L. S., Barnes, K. C., Grant, A. V., Carvalho, E. M., \& Araujo, M. I. (2012). Adult worm-specific IgE/lgG4 balance is associated with low infection levels of Schistosoma mansoni in an endemic area. Parasite Immunol, 34(12), 604-610. doi: 10.1111/pim.12001

Fitzsimmons, C. M., Falcone, F. H., \& Dunne, D. W. (2014). Helminth Allergens, Parasite-Specific IgE, and Its Protective Role in Human Immunity. Front Immunol, 5, 61. doi: 10.3389/fimmu.2014.00061

Gabrie, J. A., Rueda, M. M., Rodríguez, C. A., Canales, M., \& Sánchez, A. L. (2016). Immune Profile of Honduran Schoolchildren with Intestinal Parasites: The Skewed Response against Geohelminths. J Parasitol Res, 2016, 1769585. doi: 10.1155/2016/1769585

Lau, S., \& Matricardi, P. M. (2006). Worms, asthma, and the hygiene hypothesis. Lancet, 367(9522), 1556-1558. doi: 10.1016/S0140-6736(06)68670-4

McSorley, H. J., \& Maizels, R. M. (2012). Helminth infections and host immune regulation. Clin Microbiol Rev, 25(4), 585-608. doi: 10.1128/CMR.05040-11

Mejía Torres, R. E., Franco García, D. N., Fontecha Sandoval, G. A., Hernández Santana, A., Singh, P., Mancero Bucheli, S. T., Paz, M. Y. (2014). Prevalence and intensity of soil-transmitted helminthiasis, prevalence of malaria and nutritional status of school going children in Honduras. PLoS Negl Trop Dis, 8(10), e3248. doi: 10.1371/journal.pntd.0003248

Sanchez, A. L., Gabrie, J. A., Rueda, M. M., Mejia, R. E., Bottazzi, M. E., \& Canales, M. 
(2014). A scoping review and prevalence analysis of soil-transmitted helminth infections in Honduras. PLoS Negl Trop Dis, 8(1), e2653. doi: 10.1371/journal.pntd.0002653

WHO. (2012). Soil-transmitted helminthiases: eliminating as public health problem soil-transmitted helminthiases in children: progress report 2001-2010 and strategic plan 2011-2020. Geneva: World Health Organization.

Zaph, C., Cooper, P. J., \& Harris, N. L. (2014). Mucosal immune responses following intestinal nematode infection. Parasite Immunol, 36(9), 439-452. doi: 10.1111/pim.12090 